\title{
Identification of Wheeling Paths by an Extended Sensitivity Analysis
}

\author{
*a HIROMU HAMADA, ${ }^{\mathrm{b}}$ MARTA MARMIROLI, ${ }^{\mathrm{c}} \mathrm{RYUICHI}$ YOKOYAMA \\ ${ }^{\mathrm{a}}$ Tokyo Electric Power Company \\ 6-6 Midori-cho, Tachikawa-shi, Tokyo, 190-0014, JAPAN \\ ${ }^{\mathrm{b}}$ Mitsubishi Electric Corporation \\ 2-7-3 Marunouchi, Chiyoda-ku Tokyo, 100-8310, JAPAN \\ ${ }^{\mathrm{c}}$ Waseda University \\ 1-1-7 Nishi-Waseda, Shinjuku-ku,Tokyo, 169-0051, JAPAN \\ ahamada_tepco@yahoo.co.jp, ${ }^{\mathrm{b}}$ marmiroli.marta@bc.mitsubishielectric.co.jp, \\ cyoko@eei.metro-u.ac.jp
}

\begin{abstract}
This paper proposes a practical method for identifying wheeling paths in deregulated electricity markets based on an extended sensitivity analysis. Using this method, it becomes possible to fix the proper and fair wheeling rate according to the degree of responsibility of each power flow transaction. Moreover, a wheeling rate based on the real power flow responsibility is also an important signal to new power suppliers in the markets. In order to show the validity of the proposed method, a series of simulations on the IEEE 30 bus test system were conducted.
\end{abstract}

Key-Words: - Electricity market, Route coloring, Sensitivity analysis, Wheeling, Pricing, Deregulation

\section{Introduction}

There is a trend toward competitive markets in the electric power industry all over the world. In Japan, although the deregulation of electricity is being implemented mainly in the wholesale power market, partial retail wheeling is allowed. In other words, any party (player) who is in the business of supplying electricity to the market can supply it to its customers, not only from its own generators but also from the market, selling both inside and outside their regions. High voltage customers can purchase power from either existing regional utility companies, or new power suppliers. Since the pricing for these purchases is not regulated, bilateral contracts between suppliers and customers may be negotiated and arranged. However, if electricity based on the bilateral transactions uses the grid owned by the regional utility company, the wheeling rate must be paid to the grid owner. Under such circumstances, appropriate setting of the wheeling rate is one of the critical issues for both suppliers and grid owners in deregulated electricity markets [1].

Several methodologies to determine wheeling rates, such as postage stamp, contract path, distance based MW-mile, and power flow based MW-mile have been proposed and deeply investigated [2-4]. However, since in the aforementioned methods, the use of transmission lines and time variable load flows are not modeled sufficiently, a wheeling rate reflecting the transmission line condition (change by time and a day of week) is preferable in order to obtain a fair rate.

In this paper, we propose an efficient method for identifying wheeling paths based on an extended sensitivity analysis. Using this method, it becomes possible to fix the proper and fair wheeling rate according to the degree of responsibility of each power flow transaction. The proposed method has four advantages compared with the existing transmission routes identification techniques. (i): The proposed approach incorporates the concept of generation distribution factor [5-7] into the sensitivity analysis, which can take the consideration of not only variable loads but also generator characteristics (generation capacity, speed regulation, and dispatching strategies) and load characteristics (voltage and frequency elasticity, constant power features) under various conditions. (ii): Comparing with most of the techniques which target to identify wheeling path from single generator to single customer [8-10], the proposed method can identify multiple wheeling paths accurately, i.e., any combination among the generators and the customers, such as single to single, plurality to single, single to plurality and plurality to plurality. This approach is applicable to the situation where new power suppliers have more than two generators and there are many customers in the market. (iii): The computational complexity of 
the proposed method is approximately equivalent to one iteration of power flow calculation, and is computationally efficient. (iv) There is no special assumption, and also no additional theoretical error except numerical error.

The paper is organized as follows. In the next section, the sensitivity and generation distribution factor is described. In section 3 , in order to identify the wheeling paths of any combination among generators and customers, the sensitivity factor concept introduced in section 2 is expanded. Then, wheeling paths identification technique and the algorithm that uses the extended sensitivity method are described. In section 4 , in order to show the validity of the proposed method, several simulations on the IEEE 30-bus test system are conducted and numerical results are described. Section 5 concludes the paper.

\section{Sensitivity in Power System Operation}

\subsection{Sensitivity analysis in power systems}

To determine the sensitivity factor, firstly, is necessary to develop the sensitivity matrix of power flow [11]. The power flow of the general N-node Tbranch power system is described by a set of $\mathrm{N}$ simultaneous complex equations.

$$
P_{k}+j Q_{k}=C_{k}+j D_{k}+E_{k} \sum_{\alpha}^{N}\left(Y_{k \alpha} E_{\alpha}\right)^{*}
$$

where $P_{k}$ and $Q_{k}$ represent active and reactive power generations of the node- $k$, respectively. In addition, $C_{k}$ and $D_{k}$ represent active and reactive power demands of the node- $k$, respectively. Let the voltage vector $\boldsymbol{E}_{\boldsymbol{k}}$ at node- $k$ be expressed in polar form and the admittance $\boldsymbol{Y}_{k \alpha}$ in rectangular form. The following $2 \mathrm{~N}$ equations $\left(G_{2 k-1}=0\right.$ and $G_{2 k}=0$ $(k=1,2, \cdots, N))$ are obtained dividing (1) in the real part and the imaginary part.

$$
\begin{aligned}
& G_{2 k-1}=C_{k}-P_{k} \\
& +E_{k} \sum_{\alpha} E_{\alpha}\left\{G_{k \alpha} \cos \left(\theta_{k}-\theta_{\alpha}\right)+B_{k \alpha} \sin \left(\theta_{k}-\theta_{\alpha}\right)\right\} \\
& G_{2 k}=D_{k}-Q_{k} \\
& +E_{k} \sum_{\alpha} E_{\alpha}\left\{G_{k \alpha} \sin \left(\theta_{k}-\theta_{\alpha}\right)-B_{k \alpha} \cos \left(\theta_{k}-\theta_{\alpha}\right)\right\}
\end{aligned}
$$

For simplicity, the variables and parameters involved in (2) and (3) are defined with two vectors: (i) Dependent variable vector $\boldsymbol{X}$ (2N dimensional vector). Here, vector $\boldsymbol{X}$ comprises unknown variables in a usual power flow calculation.

(ii) Controllable variable vector $\boldsymbol{U}$ (M dimensional vector). This is a M-dimensional vector comprising the operating (manipulated) variables in system analysis and control.

Using the two vectors $\boldsymbol{X}$ and $\boldsymbol{U}$ defined above, (2) and (3) can be rewritten as the simple vector (4).

$\boldsymbol{G}(\boldsymbol{X}, \boldsymbol{U})=0$

where $\boldsymbol{G}$ is a $2 \mathrm{~N}$-dimensional column vector function with $G_{2 k-1}$ and $G_{2 k}(k=1,2, \cdots, N)$. Suppose that in a $\mathrm{N}$-node power system, the operating condition is such that $\boldsymbol{X}=\boldsymbol{X}_{\boldsymbol{0}}$ for a specified control vector $\boldsymbol{U}=\boldsymbol{U}_{\boldsymbol{0}}$. Since the pair of vectors $\boldsymbol{X}_{\boldsymbol{0}}$ and $\boldsymbol{U}_{\boldsymbol{0}}$ satisfies the power flow (4), then

$$
\boldsymbol{G}\left(\boldsymbol{X}_{\boldsymbol{0}}, \boldsymbol{U}_{\boldsymbol{0}}\right)=0
$$

Let us assume that by changing the operating condition of regulating devices (in what follows, simply called the regulating devices), the controllable variable vector $\boldsymbol{U}$ changes of a $\Delta \boldsymbol{U}$ from $\boldsymbol{U}_{\boldsymbol{0}}$. If dependent variable vector $\boldsymbol{X}$ changes from $\boldsymbol{X}_{\boldsymbol{0}}$ to $\boldsymbol{X}_{\boldsymbol{0}}+\Delta \boldsymbol{X}$ in accordance with the change $\Delta \boldsymbol{U}$, then

$$
\boldsymbol{G}\left(\boldsymbol{X}_{\boldsymbol{0}}+\Delta \boldsymbol{X}, \boldsymbol{U}_{\boldsymbol{0}}+\Delta \boldsymbol{U}\right)=0
$$

If $\Delta \boldsymbol{U}$ is taken to be very small, then the variance $\Delta \boldsymbol{X}$ is generally small. By applying Taylor's series expansion [12] to (6) with $\left(\boldsymbol{X}_{\boldsymbol{0}}, \boldsymbol{U}_{\boldsymbol{0}}\right)$ as the reference state and neglecting higher order terms in $\Delta \boldsymbol{X}$ and $\Delta \boldsymbol{U}$, we have

$\boldsymbol{G}\left(\boldsymbol{X}_{\boldsymbol{0}}, \boldsymbol{U}_{\boldsymbol{0}}\right)+\boldsymbol{G}_{\boldsymbol{X}}\left(\boldsymbol{X}_{\boldsymbol{0}}, \boldsymbol{U}_{\boldsymbol{0}}\right) \Delta \boldsymbol{X}+\boldsymbol{G}_{\boldsymbol{U}}\left(\boldsymbol{X}_{\boldsymbol{0}}, \boldsymbol{U}_{\boldsymbol{0}}\right) \Delta \boldsymbol{U}=0$ where $\boldsymbol{G}_{\boldsymbol{X}}, \boldsymbol{G}_{\boldsymbol{U}}$ are the Jacobian matrix of $\boldsymbol{G}$ with respect to dependent variable vector $\boldsymbol{X}$ and controllable variable $\boldsymbol{U}$. From (5), as the first term of (7) is zero

$\Delta \boldsymbol{X}=-\boldsymbol{G}_{X}^{-1} \cdot \boldsymbol{G}_{U} \cdot \Delta \boldsymbol{U}$

Let

$\boldsymbol{S} \equiv-\boldsymbol{G}_{\boldsymbol{X}}^{-1} \cdot \boldsymbol{G}_{\boldsymbol{U}}$

(8) can be rewritten in the form

$\Delta \boldsymbol{X}=\boldsymbol{S} \cdot \Delta \boldsymbol{U}$

or more concretely in the form

$$
\left(\begin{array}{c}
\Delta X_{1} \\
\Delta X_{2} \\
\vdots \\
\Delta X_{2 N}
\end{array}\right)=\left(\begin{array}{cccc}
S_{11} & S_{12} & \cdots & S_{1 M} \\
S_{21} & S_{22} & \cdots & S_{2 M} \\
\cdots & \cdots & \cdots & \cdots \\
S_{2 N 1} & S_{2 N 2} & \cdots & S_{2 N M}
\end{array}\right)\left(\begin{array}{c}
\Delta U_{1} \\
\Delta U_{2} \\
\vdots \\
\Delta U_{M}
\end{array}\right)
$$

The $2 \mathrm{~N} \times \mathrm{M}$ coefficient matrix $\boldsymbol{S}$ in (11) is called the sensitivity matrix of power flow with respect to the controllable variable vector $\boldsymbol{U}$.

Next, we derive the sensitivity matrix of power flow. By the use of the two vectors $\boldsymbol{X}$ and $\boldsymbol{U}$, the line flows or line currents are conveniently expressed by the simple function vector $\boldsymbol{F}(\boldsymbol{X}, \boldsymbol{U})$, where $\boldsymbol{F}$ is a T-dimensional column vector function. Let us 
assume that when the state of the system is changed from an initial state $\left(\boldsymbol{X}_{\boldsymbol{0}}, \boldsymbol{U}_{\boldsymbol{0}}\right)$ to a state $\left(\boldsymbol{X}_{0}+\Delta \boldsymbol{X}, \boldsymbol{U}_{0}+\Delta \boldsymbol{U}\right)$ by the operation of some regulating devices, line flows are also changed by $\Delta \boldsymbol{F}\left(\boldsymbol{X}_{\boldsymbol{0}}, \boldsymbol{U}_{\boldsymbol{0}}\right)$.

$\Delta \boldsymbol{F}\left(\boldsymbol{X}_{\boldsymbol{0}}, \boldsymbol{U}_{\boldsymbol{0}}\right)=\boldsymbol{F}\left(\boldsymbol{X}_{\boldsymbol{0}}+\Delta \boldsymbol{X}, \boldsymbol{U}_{\boldsymbol{0}}+\Delta \boldsymbol{U}\right)-\boldsymbol{F}\left(\boldsymbol{X}_{\boldsymbol{0}}, \boldsymbol{U}_{\boldsymbol{0}}\right)$

Like (7), the Taylor series expansion [12] of (12) with $\left(\boldsymbol{X}_{\boldsymbol{0}}, \boldsymbol{U}_{\boldsymbol{0}}\right)$ as the reference state and neglecting higher order terms in $\Delta \boldsymbol{X}$ and $\Delta \boldsymbol{U}$ yields

$$
\begin{aligned}
& \Delta F\left(X_{0}, U_{0}\right)=F\left(X_{0}, U_{0}\right)+F_{X}\left(X_{0}, U_{0}\right) \Delta X+ \\
& F_{U}\left(X_{0}, U_{0}\right) \Delta U-F\left(X_{0}, U_{0}\right)=F_{X}\left(X_{0}, U_{0}\right) \Delta X \\
& +\boldsymbol{F}_{U}\left(\boldsymbol{X}_{\boldsymbol{0}}, \boldsymbol{U}_{\boldsymbol{0}}\right) \Delta \boldsymbol{U}
\end{aligned}
$$

The sensitivity $\boldsymbol{S}_{\boldsymbol{F}}$ expresses the change $\Delta \boldsymbol{F}\left(\boldsymbol{X}_{\boldsymbol{0}}, \boldsymbol{U}_{\boldsymbol{0}}\right)$ of the power flow, which corresponds to the change $\Delta \boldsymbol{U}$ of the controllable variable vector $\boldsymbol{U}$. Hence, from (13), following relation is obtained.

$$
\begin{aligned}
& S_{F}=\frac{\Delta F\left(X_{0}, U_{0}\right)}{\Delta U} \\
& =F_{X}\left(X_{0}, U_{0}\right) \cdot \frac{\partial X}{\partial U}+F_{U} \cdot\left(X_{0}, U_{0}\right)
\end{aligned}
$$

Let $\boldsymbol{F}$ be the line power flow from node- $k$ to node- $m$. When the line power flow from node- $k$ to node- $m$ is assumed to be $P_{k m}+j Q_{k m}$, the line power flow is given by:

$$
P_{k m}+j Q_{k m}=\boldsymbol{E}_{\boldsymbol{k}}\left(\boldsymbol{E}_{\boldsymbol{k}}-\boldsymbol{E}_{\boldsymbol{m}}\right)^{*}\left(-\boldsymbol{Y}_{\boldsymbol{k} m}\right)^{*}
$$

Thus, $\boldsymbol{F}(\boldsymbol{X}, \boldsymbol{U})$ is given by following general form.

$$
\boldsymbol{F}(\boldsymbol{X}, \boldsymbol{U}) \equiv \boldsymbol{F}\left(E_{k}, E_{m}, \boldsymbol{\theta}_{k}, \theta_{m}, \boldsymbol{U}\right)
$$

Partial derivatives, $\boldsymbol{F}_{\boldsymbol{X}}$ and $\boldsymbol{F}_{\boldsymbol{U}}$ are obtained quite easily by simple calculation. $(\partial \boldsymbol{X} / \partial \boldsymbol{U})$, i.e., $\partial E_{k} / \partial \boldsymbol{U}, \partial E_{m} / \partial \boldsymbol{U}, \partial \theta_{k} / \partial \boldsymbol{U}$ and $\partial \theta_{m} / \partial \boldsymbol{U}$ are the sensitivities of $E_{k}, E_{m}, \theta_{k}$ and $\theta_{m}$ to the unit amount of change in a regulating device, which is already known as the elements of the sensitivity matrix $\boldsymbol{S}$. Thus the sensitivity constants for line power flows and line currents can be calculated from (14).

\subsection{Generation distribution factor}

Next, we describe generation distribution factor $[5,11]$, which plays an important role in identifying wheeling paths for the cases with two or more wheeling contracts. The possibility to identify wheeling paths for multiple contracts is an important advantage of the proposed method.

Generation distribution factor represents the effect of a one-per-unit change in the output of a generator on line currents and line power flows. This factor is also a useful measure for generation shifting in preventive and emergency control. In generation shifting, the power balance between generator outputs must be maintained. Policy of maintaining power balance in the system is incorporated using redistribution coefficients defined as follows:

$$
\Delta P_{l m}=-\frac{P_{R_{l}} \cdot \Delta P_{m}}{R_{l} \cdot f_{0} \cdot \sum_{\substack{i \\ i \neq m}} \frac{P_{R_{i}}}{R_{i} \cdot f_{0}}}=-K_{l m} \cdot \Delta P_{m}
$$

Here, the constraint on power balance is described by:

$\Delta P_{m}=\sum_{\substack{i \\ i \neq m}}^{L} \Delta P_{i m}$

where $\Delta P_{l m}$ is the change of the active power of generator $l$-th caused by the change of the active power $\Delta P_{m}$ of generator $m$-th; $P_{R_{i}}$ is the rated capacity of the $i$-th generator; $R_{i}$ is the speed regulation of the $i$-th generator; $f_{0}$ is the rated frequency.

$K_{l m}$ is referred to as the redistribution coefficient. When generation shifting is determined by (17), the imbalance of real power is redistributed in accordance with each generator characteristic, e.g., capacity, speed regulation, etc. In (17), if the speed regulation of each generator is the same, the imbalance is redistributed proportionally to each generator capacity. Furthermore by assigning 1 to a particular $K_{l m}$, the $l$-th generator is intended as the slack generator.

\section{Identifying Wheeling Paths by an Extended Sensitivity Method}

While the method described in section 2.1 only identifies wheeling path between single generator and single customer [13], by incorporating generation distribution factor described in section 2.2 into sensitivity, the proposed method can identify multiple wheeling paths accurately, i.e., any combination among the generators and the customers, such as single to single, plurality to single, single to plurality and plurality to plurality. In this section, firstly by extending the sensitivity method described in section 2.1, we describe how to incorporate the characteristics of power system. Next, we describe how to incorporate also generation distribution factor in the sensitivity analysis. Finally, the method of identifying wheeling paths and the algorithm to solve it are described. 


\subsection{Sensitivity matrix including system characteristics}

In the extended sensitivity method proposed here, generator characteristics (generation capacity, speed regulation, and dispatching strategies) and load characteristics (voltage, frequency elasticity and constant power features) can be taken into account by modifying the sensitivity matrix in the process of Jacobian derivations. Inclusion of system characteristics requires the modification of the power flow equation expressed by (4). Let the power flow of the general power system be described by the simple vector equation.

$\boldsymbol{G}(\boldsymbol{X}, \boldsymbol{U}, \phi(\boldsymbol{X}), \beta(\boldsymbol{U}))=0$

where $\phi(X)$ is $2 \mathrm{~N}$-dimensional vector function of $\boldsymbol{X} ; \beta(\boldsymbol{U})$ is $2 \mathrm{~N}$-dimensional vector function of $\boldsymbol{U}$. Vector $\boldsymbol{U}$ and $\boldsymbol{X}$ are the same as defined in section 2 . By the introduction of the functions $\phi(X)$ and $\beta(\boldsymbol{U})$, defined anew, various kinds of system characteristics in the power system can be modeled. As in (7), suppose that the dependent variable vector $\boldsymbol{X}$ changes from $\boldsymbol{X}_{0}$ to $\boldsymbol{X}_{0}+\Delta \boldsymbol{X}$ in accordance with the change of controllable vector by $\Delta \boldsymbol{U}$, then

$$
\frac{\partial \boldsymbol{G}}{\partial \boldsymbol{X}} \Delta \boldsymbol{X}+\frac{\partial \boldsymbol{G}}{\partial \boldsymbol{U}} \Delta \boldsymbol{U}+\frac{\partial \boldsymbol{G}}{\partial \phi} \cdot \frac{\partial \phi}{\partial \boldsymbol{X}} \Delta \boldsymbol{X}+\frac{\partial \boldsymbol{G}}{\partial \beta} \cdot \frac{\partial \beta}{\partial \boldsymbol{U}} \Delta \boldsymbol{U}=0
$$

Therefore, the sensitivity matrix of the power system expressed by (19) is given by:

$$
\boldsymbol{S}_{\boldsymbol{m}}=\frac{\partial \boldsymbol{X}}{\partial \boldsymbol{U}}=-\left(\frac{\partial \boldsymbol{G}}{\partial \boldsymbol{X}}+\frac{\partial \boldsymbol{G}}{\partial \phi} \cdot \frac{\partial \phi}{\partial \boldsymbol{X}}\right)^{-1} \cdot\left(\frac{\partial \boldsymbol{G}}{\partial \boldsymbol{U}}+\frac{\partial \boldsymbol{G}}{\partial \beta} \cdot \frac{\partial \beta}{\partial \boldsymbol{U}}\right)
$$

The Jacobian matrices $\boldsymbol{G}_{\boldsymbol{X}}$ and $\boldsymbol{G}_{\boldsymbol{U}}$ are computed in the same way as in (9). On the other hand, $\frac{\partial \boldsymbol{G}}{\partial \phi} \cdot \frac{\partial \phi}{\partial \boldsymbol{X}}$ and $\frac{\partial \boldsymbol{G}}{\partial \beta} \cdot \frac{\partial \beta}{\partial \boldsymbol{U}}$ are modification terms caused by introducing the functions $\phi(\boldsymbol{X})$ and $\beta(U)$ into the power flow (19). It is obvious from (21) that if the first derivatives of functions $\phi$ and $\beta$ with respect to $\boldsymbol{X}$ and $\boldsymbol{U}$ exist, these modification terms can be computed easily.

(21) is the fundamental equation for determining various basic data to be used in system operation. Each basic data is obtained in a systematic way from the modified sensitivity matrix by simply changing the modification terms representing the system characteristics.

\subsection{Power flow modification}

When generation distribution factor is computed, minor modifications of power system equations are necessary by introducing coefficient $K_{l m}$. In the generation shifting, the control variable is $\Delta P_{m}$, the change in $m$-th generator output. Power flow equations for each node can be modified as follows.

(i) Power flow equations for $m$-th demand node

$G_{2 m-1}=C_{m}-\left(P_{m}+\Delta P_{m}\right)$

$+E_{m} \sum_{\alpha} E_{\alpha}\left\{G_{m \alpha} \cos \left(\theta_{m}-\theta_{\alpha}\right)+B_{m \alpha} \sin \left(\theta_{m}-\theta_{\alpha}\right)\right\}$

$G_{2 m}=D_{m}+Q_{m s e t}$

$+E_{m} \sum_{\alpha} E_{\alpha}\left\{G_{m \alpha} \sin \left(\theta_{m}-\theta_{\alpha}\right)-B_{m \alpha} \cos \left(\theta_{m}-\theta_{\alpha}\right)\right\}$

(ii) Power flow equations for $l$-th generator node

$G_{2 l-1}=C_{l}-\left(P_{\text {lset }}-K_{l m} \cdot \Delta P_{m}\right)$

$+E_{l} \sum_{\alpha} E_{\alpha}\left\{G_{l \alpha} \cos \left(\theta_{l}-\theta_{\alpha}\right)+B_{l \alpha} \sin \left(\theta_{l}-\theta_{\alpha}\right)\right\}$

$G_{2 l}=D_{l}-Q_{l s e t}$

$+E_{l} \sum_{\alpha} E_{\alpha}\left\{G_{l \alpha} \sin \left(\theta_{l}-\theta_{\alpha}\right)-B_{l \alpha} \cos \left(\theta_{l}-\theta_{\alpha}\right)\right\}$

(iii) Power flow equations for other nodes

$G_{2 k-1}=C_{k}-P_{k}$

$+E_{k} \sum_{\alpha} E_{\alpha}\left\{G_{k \alpha} \cos \left(\theta_{k}-\theta_{\alpha}\right)+B_{k \alpha} \sin \left(\theta_{k}-\theta_{\alpha}\right)\right\}$

$G_{2 k}=D_{k}-Q_{k}$

$+E_{k} \sum_{\alpha} E_{\alpha}\left\{G_{k \alpha} \sin \left(\theta_{k}-\theta_{\alpha}\right)-B_{k \alpha} \cos \left(\theta_{k}-\theta_{\alpha}\right)\right\}$

where $P_{s e t}, Q_{\text {set }}$ are specified real and reactive power generations. Note that $P_{l}$ is an element for the vector of operation variables $\boldsymbol{U}$. The Jacobian matrix $\boldsymbol{G}_{\boldsymbol{U}}$ of $\boldsymbol{U}$ is different from $\boldsymbol{G}_{\boldsymbol{U}}$ of section 2, but the Jacobian matrix $\boldsymbol{G}_{\boldsymbol{X}}$ for the dependent variables is the same as $\boldsymbol{G}_{\boldsymbol{X}}$ of section 2 .

From the above-mentioned quotation, by using the sensitivity equation expressed by (11), generation distribution factor can be obtained. Also, $\beta(\boldsymbol{U})$ of (19) corresponds to $-K_{l m} \cdot \Delta P_{m}$ of (24) and $\frac{\partial G}{\partial \boldsymbol{\beta}} \cdot \frac{\partial \boldsymbol{\beta}}{\partial U}$ of (21) corresponds to $K_{l m}$.

\subsection{Algorithm}

The sensitivity matrix for identifying the wheeling paths among any combination of generators and power consumers, including multiple transaction, can be obtained according to the following algorithm.

The proposed method can obtain the sensitivities necessary for identifying the wheeling route by calculating generation distribution factor. Specifically, we compute the generation distribution factor by letting (22) and (23) represent the 
customer (the $m$-th demand node) and letting (24) and (25) represent the generator (the $l$-th generator node).

(i) Wheeling from single generator to single customer.

Firstly, when there is only one generator for wheeling, let the power flow equations of the generator be (24) and (25), where $K_{l m}=1$. Other nodes follow (22), (23), (26) and (27). Then, by using (21), we can obtain the sensitivities necessary for identifying the wheeling route, by calculating generation distribution factor.

(ii) Wheeling from plural generators to single customer

When there are plural generators for wheeling, the value of redistribution coefficient $K_{l m}$ is selected according to the ratio of power outputs among generators to calculate the generation distribution factor by using (21).

By the setting method of $K_{l m}$, it becomes possible to model the generation dispatching strategies.

For example, assume that the power supplier has two generators A and B, which supply electricity of wheeling $60 \%$ and $40 \%$ respectively. The power flow equations of the generators A and B are (24) and (25), where $K_{l m}$ are 0.6 and 0.4 respectively. The power flow equations for other nodes are (22), (23), (26) and (27). With those equations and (21), we can obtain the sensitivities by calculating generation distribution factor in a similar manner. On the other hand, when the outputs of generators are decided by their $P_{R_{i}}$ and $R_{i}, K_{l m}$ is calculated based on (17) and (18). Therefore, the sensitivities can be obtained by (22)-(27) and (21).

(iii) Wheeling from single generator to plural customers and plural generators to plural customers In the case of wheeling to plural customers, the linearity and the superposition are utilized $[11,14]$. The related wheeling path is identified easily by investigating the magnitude of sensitivity factors calculated by the above-mentioned procedure.

For instance, assume that there are two locations C and $\mathrm{D}$ for a customer, which consume the electricity $60 \%$ and $40 \%$ respectively. Firstly, depending on single or plural generators, (i) or (ii) is used to calculate the sensitivities represented by $\mathrm{C}$ and $\mathrm{D}$ respectively. Then, the total sensitivity is $0.6 \mathrm{C}+0.4 \mathrm{D}$. Based on the above discussion, the algorithm to identify wheeling paths can be summarized as follows.

[Step 1] Power Flow: Power flow calculation.

[Step 2]Construct $\boldsymbol{G}_{\boldsymbol{X}}$ : By modifying the Jacobian matrix of power flow equations, construct
$\boldsymbol{G}_{\boldsymbol{X}}$ which is the Jacobian matrix with respect to $X$.

[Step 3]Multiple wheeling paths: Go to [step4] for wheeling paths of multiple consumers; go to [step5] for wheeling path of single consumer.

[Step 4]Decompose wheeling: When the wheeling is from single generator to multiple consumers, such wheeling is calculated by summing all of single-generator to single-consumer cases. When the wheeling is from multiple generators to multiple consumers, such wheeling is calculated by summing all of multiple-generator to single-consumer cases.

[Step 5]Construct $\boldsymbol{G}_{\boldsymbol{U}}$ : Construct the Jacobian matrix with respect to the controllable variable $\boldsymbol{U}$. For single generator, $K_{l m}$ is equal 1. For multiple generators, $K_{l m}$ is set with proportionally to the output of generators.

[Step 6]Construct the sensitivity matrix: By $\boldsymbol{G}_{\boldsymbol{X}}$ and $\boldsymbol{G}_{U}$, construct sensitivity matrix $\boldsymbol{S}_{\boldsymbol{m}}$ from (21).

[Step 7]Construct the sensitivities for active power flow: Calculate line power flow based on the sensitivity matrix of [step6]. In case of wheeling to single consumer, go to [step9]; in case of wheeling to multiple consumers, go to [step8].

[Step 8]Superposition of the wheeling: Proportional to load ratios of consumers, superpose wheeling of [step7]. Calculate the power flow sensitivities.

[Step 9]Identify wheeling paths: Based on the power flow sensitivities, identify wheeling paths. For instance, if the sensitivity of the line $\mathrm{E}$ is 0.25 calculated by the algorithm, the power flow in the line $\mathrm{E}$ is $25 \%$ of total wheeling.

Hence, based on the algorithm, we can identify the wheeling on each line.

\section{Application of Identifying Wheeling Paths to a Test System \\ 4.1 Configuration and characteristics of a test system}

In this section, the sensitivity-based method is applied to a test system to identify wheeling paths in transmission networks. The structure of the IEEE 30-bus system with 19 loads, 4 generators and 41 transmission lines are shown in Fig. 1, which is used as a demonstration test system in this paper. 
In order to create a variety of wheeling paths among suppliers and customers, rotary condensers at nodes 5 and 8 in the original test system are replaced by generators. The direction of arrows in the figure shows the direction of the power flow at a base load condition.

A set of wheeling from nodes 1 and 2 at $132 \mathrm{KV}$ buses to nodes 16 and 21 at $33 \mathrm{KV}$ buses has been assumed. Comparisons regarding the accuracy and the amount of calculations for the sensitivity of the proposed approach and the precise calculations have been carried out, and also changes of the sensitivity by different load conditions and identification of wheeling paths have been investigated.

\subsection{Sensitivity by repetitive power flow calculation}

In this section, we describe an iterative numerical method for the computation of sensitivities [10] that we want to compare numerically with our proposed method. Since the purpose of wheeling path identification is to set the wheeling prices, a high accuracy in the results is required. The proposed method is accurate due to the exact power flow calculation (theoretical value can also be derived). In addition, we do not require any specific assumption, and there is no theoretical error due to modeling approximations.

In the iterative method, the sensitivity is defined as follows.

$$
\begin{aligned}
& \Delta P_{k}=S_{k j} \Delta P_{j} \\
& \boldsymbol{S}=\frac{\boldsymbol{P}_{N E \boldsymbol{W}}-\boldsymbol{P}_{\text {oLD }}}{\Delta P}
\end{aligned}
$$

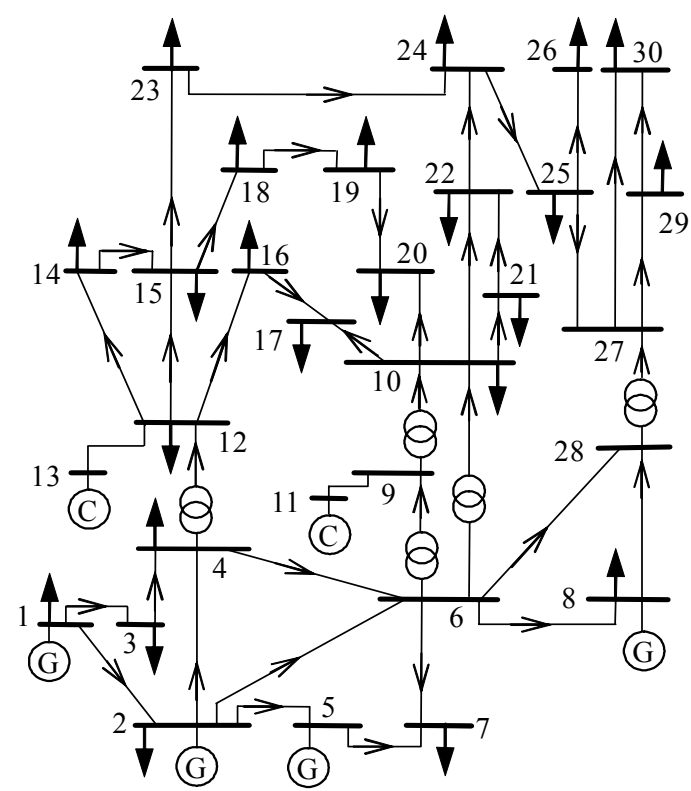

Fig. 1 IEEE 30 node test system where $\boldsymbol{P}_{\boldsymbol{O} L \boldsymbol{D}}$ denotes the load profile at the base case and $\boldsymbol{P}_{N E W}$ is the power flow after slightly changing a control variable $\Delta P$.

\subsection{Comparison of accuracy}

In this section, we compare the proposed algorithm with the iterative method described above.

For the assumed wheeling from nodes 1 and 2 to nodes 16 and 21, as shown in Table 2, sensitivity factors have been calculated by the proposed extended sensitivity method (ESM) and the above mentioned repetitive power flow calculation method (PFM). The correspondence of branch numbers and node numbers illustrated in Fig. 1 is shown in Table 1.

According to Table 2, clearly the relative error of sensitivity by the proposed method against the exact power flow calculation is within $1.0 \%$ of the total amount of wheeling. Notice that, the sensitivity calculated in TABLE 2 is for the wheeling between two generators and two demands, which can be evaluated by the conventional sensitivity method shown in (21). In contrast to the conventional approaches (2), (3) and (9) which mainly find the wheeling path for a single contract, the proposed method can identify wheeling paths among plural generators and plural customers.

\subsection{Comparison of computational time}

In this section, we compare the proposed algorithm with the iterative method of Section 4.2 from the CPU time point of view.

In the repetition of power flow calculation method, the larger the number of wheeling is, the larger we must repeat the power flow computations, the iteration of power flow calculation for obtaining

\begin{tabular}{cccccc}
\multicolumn{6}{c}{ Table 1 Correspondence of Branches and Nodes } \\
\hline branch & node & branch & node & branch & node \\
\hline 1 & $1-2$ & 15 & $4-12$ & 29 & $21-22$ \\
2 & $1-3$ & 16 & $12-13$ & 30 & $15-23$ \\
3 & $2-4$ & 17 & $12-14$ & 31 & $22-24$ \\
4 & $3-4$ & 18 & $12-15$ & 32 & $23-24$ \\
5 & $2-5$ & 19 & $12-16$ & 33 & $24-25$ \\
6 & $2-6$ & 20 & $14-15$ & 34 & $25-26$ \\
7 & $4-6$ & 21 & $16-17$ & 35 & $25-27$ \\
8 & $5-7$ & 22 & $15-18$ & 36 & $28-27$ \\
9 & $6-7$ & 23 & $18-19$ & 37 & $27-29$ \\
10 & $6-8$ & 24 & $19-20$ & 38 & $27-30$ \\
11 & $6-9$ & 25 & $10-20$ & 39 & $29-30$ \\
12 & $6-10$ & 26 & $10-17$ & 40 & $8-28$ \\
13 & $9-11$ & 27 & $10-21$ & 41 & $6-28$ \\
14 & $9-10$ & 28 & $10-22$ & & \\
\hline
\end{tabular}


Table 2 Comparison of Sensitivity Constants by Different Approaches

\begin{tabular}{crrr}
\hline branch & \multicolumn{1}{c}{ ESM } & \multicolumn{1}{c}{ PFM } & error [\%] \\
\hline 1 & -0.0514 & -0.0511 & 0.48 \\
2 & 0.2481 & 0.2485 & 0.13 \\
3 & 0.2988 & 0.2989 & 0.02 \\
4 & 0.2420 & 0.2413 & 0.27 \\
5 & 0.1528 & 0.1528 & 0.01 \\
6 & 0.3414 & 0.3414 & 0.01 \\
7 & 0.2060 & 0.2057 & 0.13 \\
8 & 0.1494 & 0.1493 & 0.06 \\
9 & -0.1493 & -0.1494 & 0.01 \\
10 & 0.0199 & 0.0198 & 0.38 \\
11 & 0.3736 & 0.3737 & 0.02 \\
12 & 0.2087 & 0.2088 & 0.03 \\
13 & 0 & 0 & 0 \\
14 & 0.3737 & 0.3738 & 0.02 \\
15 & 0.3255 & 0.3255 & 0.01 \\
16 & 0 & 0 & 0 \\
17 & 0.0225 & 0.0226 & 0.42 \\
18 & 0.0894 & 0.0895 & 0.06 \\
19 & 0.2038 & 0.2040 & 0.09 \\
20 & 0.0221 & 0.0220 & 0.40 \\
21 & 0.0118 & 0.0118 & 0.03 \\
22 & 0.0518 & 0.0519 & 0.12 \\
23 & 0.0510 & 0.0510 & 0.03 \\
24 & 0.0510 & 0.0508 & 0.21 \\
25 & -0.0524 & -0.0526 & 0.39 \\
26 & -0.0118 & -0.0119 & 0.72 \\
27 & 0.4677 & 0.4674 & 0.06 \\
28 & 0.1781 & 0.1780 & 0.09 \\
29 & -0.3367 & -0.3370 & 0.07 \\
30 & 0.0576 & 0.0575 & 0.14 \\
31 & -0.1596 & -0.1597 & 0.05 \\
32 & 0.0572 & 0.0572 & 0.10 \\
33 & -0.1006 & -0.1006 & 0.05 \\
34 & 0 & 0 & 0 \\
35 & -0.1017 & -0.1018 & 0.15 \\
36 & 0.1031 & 0.1031 & 0.03 \\
37 & 0 & 0 & 0 \\
38 & 0 & 0 & 0 \\
39 & 0 & 0 & 0 \\
40 & 0.0212 & 0.0213 & 0.55 \\
41 & 0.0817 & 0.0818 & 0.09 \\
\hline
\end{tabular}

ESM: Sensitivity constants obtained by Extended Sensitivity Method PFM: Sensitivity constants obtained by Power Flow Method

sensitivities increases as represented by Fig. 2. The number of power flow calculation with convergence adds up to the sum of multiplication of the total combination of generators $\left(2^{m}\right)$ and the number of busses minus the number of generators $(n-m)$ and one base case calculation, so $2^{m} \times(n-m)+1$ power flow calculations are necessary for obtaining all the sensitivities for m-generators $n$-nodes power system. As indicated in Fig. 2, numerous power flow calculations have to be executed for large-scale power systems including many generators.

On the other hand, it is sufficient to execute only one power flow calculation for obtaining all the sensitivities to identify each wheeling path among generators and customers for any combination when we use the proposed extended sensitivity method. Also, if generation dispatching strategies of power producer have been determined, all the sensitivities to identify each wheeling path from the power producer to all customers can be obtained by one calculation of (21). That is to say, by one power flow calculation and $2^{m}$ calculations of (21), all the sensitivities required for identifying all wheeling paths for any combination can be obtained for $\mathrm{m}$ generators n-nodes power system. Here, (21) is not repetitive a calculation unlike power flow calculation.

In addition, in the actual present Japanese electrical power system, although the number of $n$ is over several thousands, the number of $\mathrm{m}$ is less than 10 , where $m$ represents the number of power suppliers. On the other hand, the computational complexity of the proposed method does not depend on the number of nodes $\mathrm{n}$. Therefore, the proposed method is much efficient even for a large scale system. In this paper, we compare the computational time by numerical simulation. We adopt Pentium III $800 \mathrm{HZ}$ CPU by using MATLAB. To identify wheeling paths from a single-generator to all consumers, CPU time of ESM (the proposed method) is $1.262 \mathrm{sec}$., in contrast to $24.535 \mathrm{sec}$. of PFM described in Section 4.2 , which verifies the efficiency of the proposed method.

\subsection{Comparison of the sensitivity by different load conditions}

In this section, we compare the sensitivities on different conditions of loads, i.e. light load, standard load and heavy load. The results are shown in Table

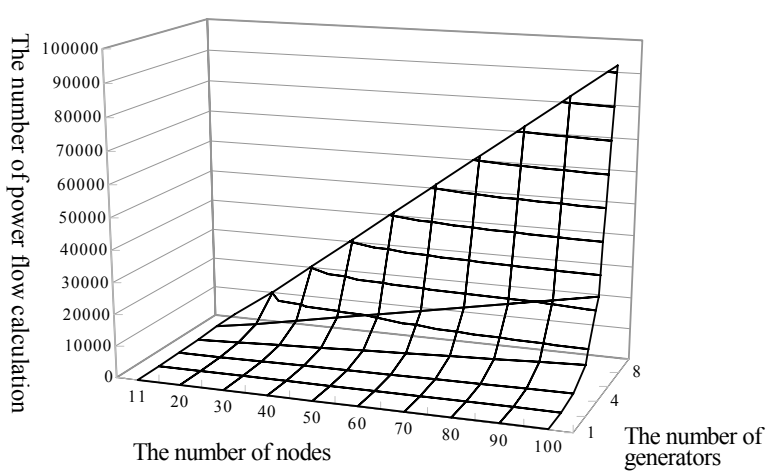

Fig. 2 Comparison of computation burden for sensitivity coefficients 
Table 3 Comparison of sensitivity constants in different load conditions

\begin{tabular}{crrr}
\hline branch & \multicolumn{1}{c}{ light } & middle & \multicolumn{1}{c}{ peak } \\
\hline 1 & -0.0585 & -0.0514 & -0.0438 \\
2 & 0.2443 & 0.2481 & 0.2523 \\
3 & 0.2968 & 0.2988 & 0.3015 \\
4 & 0.2410 & 0.2420 & 0.2455 \\
5 & 0.1506 & 0.1528 & 0.1550 \\
6 & 0.3385 & 0.3414 & 0.3446 \\
7 & 0.2050 & 0.2060 & 0.2057 \\
8 & 0.1488 & 0.1494 & 0.1514 \\
9 & -0.1478 & -0.1493 & -0.1504 \\
10 & 0.0209 & 0.0199 & 0.0206 \\
11 & 0.3725 & 0.3736 & 0.3752 \\
12 & 0.2088 & 0.2087 & 0.2087 \\
13 & 0 & 0 & 0 \\
14 & 0.3724 & 0.3737 & 0.3752 \\
15 & 0.3239 & 0.3255 & 0.3282 \\
16 & 0 & 0 & 0 \\
17 & 0.0222 & 0.0225 & 0.0230 \\
18 & 0.0886 & 0.0894 & 0.0908 \\
19 & 0.2028 & 0.2038 & 0.2055 \\
20 & 0.0218 & 0.0221 & 0.0224 \\
21 & 0.0115 & 0.0118 & 0.0125 \\
22 & 0.0520 & 0.0518 & 0.0522 \\
23 & 0.0513 & 0.0510 & 0.0518 \\
24 & 0.0513 & 0.0510 & 0.0518 \\
25 & -0.0519 & -0.0524 & -0.0526 \\
26 & -0.0114 & -0.0118 & -0.0125 \\
27 & 0.4669 & 0.4677 & 0.4698 \\
28 & 0.1780 & 0.1781 & 0.1787 \\
29 & -0.3365 & -0.3367 & -0.3389 \\
30 & 0.0578 & 0.0576 & 0.0580 \\
31 & -0.1599 & -0.1596 & -0.1598 \\
32 & 0.0572 & 0.0572 & 0.0575 \\
33 & -0.1013 & -0.1006 & -0.0999 \\
34 & 0 & 0 & 0 \\
35 & -0.1026 & -0.1017 & -0.1014 \\
36 & 0.1035 & 0.1031 & 0.1032 \\
37 & 0 & 0 & 0 \\
38 & 0 & 0 & 0 \\
39 & 0 & 0 & 0 \\
40 & 0.0210 & 0.0212 & 0.0215 \\
41 & 0.0826 & 0.0817 & 0.0815 \\
\hline & & &
\end{tabular}

3 , where the light load and heavy load are set as $80 \%$ and $120 \%$ of the standard load, respectively. Here, the choice of light and heavy load $(80 \%$ and $120 \%$ ) is based on the Japanese daily load curve.

According to Table 3, the sensitivities are different depending on load conditions. Previously developed methodologies [8-10] where not able to individuate the sensitivity difference due to a different load condition, on opposite the proposed method is able to catch the influence of the load on the sensitivities as shown in Table 3.

\subsection{Wheeling Paths}

In this section, we will identify the wheeling paths for the example described in 4.1. As described, the intent is to identify the wheeling path for transactions from nodes 1 and 2 to nodes 16 and 21 . The rate of generation in nodes 1 and 2 may be determined using a simple ELD algorithm. It is assumed that the rate of wheeling between nodes 16 and 21 is $2: 8$. Since sensitivity may be assumed to be linear [11,14] for each result $A$ and $B$, $0.2 \mathrm{~A}+0.8 \mathrm{~B}$ will become the sensitivity for this numerical example.

Sensitivities based on the proposed extended sensitivity method for wheeling from nodes 1 and 2 to nodes 16 and 21 are shown in Fig. 3 and the results of identifying wheeling paths are illustrated in Fig. 4. Oriented arrows in Fig. 4 indicate wheeling paths having sensitivities higher than 0.2. The sign of sensitivities in Fig. 3 is positive when the power flow increases by increase of a specific generator output, and the correspondence of branch numbers in Fig. 3 is shown in Table 1.

As we can see regarding wheeling from nodes 1 and 2 to nodes 16 and 21 in Fig. 4, for node 16, main stream for this wheeling is a power flow going through nodes 1-3-4 and nodes 2-4, which merge into one at node 4 and finally those are passing through nodes 4-12-16. On the other hand, for node 21 , main stream for this wheeling is a power flow going through nodes 1-3-4 and nodes 2-4, which merge into one at node 4 , then a power flow going through nodes 4-6 and nodes 2-6, which merge into one at node 6 , and passing along nodes 6-9-10 and 6-10 transformer and finally those are passing through nodes $10-21.46 .8 \%$ of power flow for this wheeling is passing through this path. The other stream is a power flow going through nodes 10-22 and nodes $24-22$, which merge into one at node 22

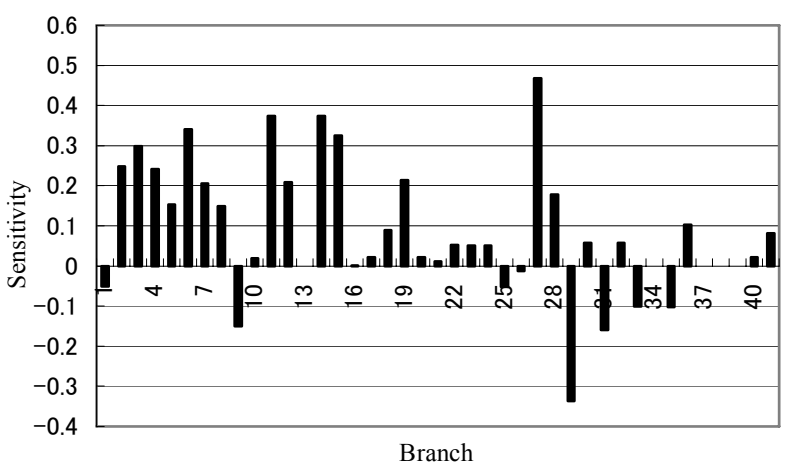

Fig. 3 Sensitivities of power flows by wheeling among nodes 1,2 and 16,21 




Fig. 4 Paths of wheeling by wheeling among nodes 1,2 and 16,21

and finally those are passing through nodes 22-21. From the example, it is evident that the sensitivity for each branch can be calculated for any combination of generation-load transactions with only one calculation and not four (number of total transaction: node1-16, node1-21, node2-16, node221 ) as with a conventional power flow sensitivity approach.

\section{Conclusions}

In this paper, we proposed a method to identify the wheeling paths by an extended sensitivity analysis. We show that it is possible to identify wheeling paths in any situation effectively by making use of the sensitivities calculated by this method. There is no limitation for the combination of generators and customers, such as single to single, plurality to single, single to plurality and plurality to plurality. To verify the effectiveness of the proposed method, several simulations have been conducted on the IEEE 30-node test system. The results of applications have clarified that the proposed method enables us to decide the path of any wheeling for any combination of market players in the system easily and accurately. In the future, the authors intend to apply the described method to the wheeling charges calculation problem under several market design combinations.
References:

[1] D. Hirano and K. Yamaji, Estimation of Longterm Wheeling Rate Using a Dynamic Model, Trans. IEE Jpn., Vol.117, 1997, pp. 33-40.

[2] R. Yokoyama, H. Asano, J. Arai, K. Okada, J. Kubokawa, I. Kurihara, et al., Liberalization of electricity markets and technological issues, Tokyo Denki University Press, 2001.

[3] D. Shirmohammadi, X. V. Filho, B. Gorenstin, and M. Pereira, Some Fundamental Technical Concepts about Cost Based Transmission Pricing, IEEE Trans. Power Syst., Vol.11, 1996, pp. 1002-1008.

[4] S. Phichaisawat and Y. H. Song, Transmission Pricing Using Improved Sensitivity Indices, IEEE Power Engineering Society Winter Meeting, Vol.3, 2001, pp. 1250-1255.

[5] B. M. Weedy and B. J. Cory, Electric power systems, Wiley, 1998.

[6] J. W. Bialek, Topological generation and load distribution factors for supplement charge allocation in transmission open access, IEEE Trans. Power Syst., Vol.12, 1997, pp. 11851193.

[7] R. Baldick, Variation of distribution factors with loading, IEEE Trans. Power Syst., Vol.18, 2003, pp. 1316-1323.

[8] D. Kirschen and G. Strbac, Tracing active and reactive power between generators and loads using real and imaginary currents, IEEE Trans. Power Syst., Vol.14, 1999, pp. 1312-1319.

[9] J. Bialek, Tracing the flow of electricity, IEE Proc. Gener., Transm., and Distrib., Vol.143, 1996, pp. 313-320.

[10] T. Ogawa, K. Tanaka, and T. Nagao, Classifying the Power Flow by Each Generator, Central Research Institute of Electric Power Industry, 1985.

[11] R. Yokoyama, S. Narita, and Y. Tamura, Security Monitoring and Control in Power System Operation, Waseda University, 1973.

[12] H. Sugie, A.Okazaki, Y. Adachi, and M. Ozaki, Numerical analysis by FORTRAN 77, Baifukan, 1999.

[13] H. Hamada, R. Yokoyama, L. Chen and T. Asano, Coloring Transmission Route for Wheeling by a Modified Sensitivity Analysis in Competitive Power Markets, 2003 National Convention IEE Jpn., Vol.6, 2003, pp. 229-230.

[14] E. Shimoda, T. Ohtaka and S. Iwamoto, Power Flow Tracing using Power Flow Equations, 2004 National Convention IEE Jpn., Vol.6, 2004, pp. 95-96. 